\title{
Culture de la qualite et culture du projet, au sein d'une société communicationnelle, enjeux et paradoxes
}

Jean-Pierre Boutinet

\section{(2) OpenEdition Journals}

Édition électronique

URL : http://journals.openedition.org/communicationorganisation/2174

DOI : 10.4000/communicationorganisation.2174

ISSN : $1775-3546$

Éditeur

Presses universitaires de Bordeaux

Édition imprimée

Date de publication : 1 mai 1999

ISSN : 1168-5549

Référence électronique

Jean-Pierre Boutinet, «Culture de la qualite et culture du projet, au sein d'une société

communicationnelle, enjeux et paradoxes », Communication et organisation [En ligne], 15। 1999, mis en ligne le 26 mars 2012, consulté le 19 avril 2019. URL : http://journals.openedition.org/ communicationorganisation/2174 ; DOI : 10.4000/communicationorganisation.2174

Ce document a été généré automatiquement le 19 avril 2019

(c) Presses universitaires de Bordeaux 


\title{
Culture de la qualite et culture du projet, au sein d'une société communicationnelle, enjeux et paradoxes
}

\author{
Jean-Pierre Boutinet
}

1 Les pratiques organisationnelles autour des dispositifs qualité et conduites de projet ont connu les unes et les autres une montée en puissance ces dernières décennies au fur et à mesure d'ailleurs que se développait notre civilisation communicationnelle. Ces pratiques sont devenues tellement incontournables qu'elles ont contribué à engendrer chacune une véritable culture, faisant que désormais, nous pensons spontanément les réalisations que nous menons par référence à la qualité et au projet. Cette montée en puissance simultanée nous interroge donc sur les relations de convergence ou de conflit que ces deux concepts apparemment étrangers l'un à l'autre entretiennent entre eux: les pratiques centrées sur les conduites à projet, s'inscrivent-elles dans la même logique que les pratiques développées autour de la qualité ? Quelles convergences, quels paradoxes relient ces pratiques et de ce fait sont susceptibles de mieux éclairer la culture communicationnelle à laquelle projet et qualité semblent l'un et l'autre attachés? Pour répondre à de telles questions nous nous situerons principalement sur les plans historique et méthodologique de l'évolution et de l'organisation des pratiques concernées.

\section{Les conduites à projet et leur dimension communicationnelle}

Parler de conduites à projet c'est évoquer une pluralité de figures : projet d'entreprise, projet de formation, projet professionnel, gestion ou management par projet $^{1}$. Certes le projet d'entreprise après des hauts et des bas se trouve actuellement bien mal en point 
dans une période où règne le downsizing et le re engineering. Nous le laisserons donc de côté ; nous nous intéresserons en revanche aux trois autres figures qui occupent toujours le devant de l'actualité, surtout la dernière, la gestion de ou par projet; celle-ci en un demi-siècle s'est progressivement promue sur l'avant scène des pratiques gestionnaires et sociotechniques.

Ces figures du projet professionnel, du projet de formation et de la gestion de projet telles qu'elles se sont imposées de plus en plus massivement mettent en jeu la prééminence d'un acteur individuel ou collectif qui élabore des scénarios, explicite des intentions en réponse à un appel d'offre, anticipe, décide; il le fait en inscrivant son action dans des temporalités bien déterminées, forcément discontinues parce que liées à la durée de la mission nécessaire pour l'implantation d'un projet. Ces temporalités ouvrent donc sur l'éphémère et l'obsolescence puisque l'innovation incarnée par n'importe quel projet est très souvent destinée à être supplantée par une innovation subséquente. Avec la généralisation des pratiques de projet, telles que nous les connaissons aujourd'hui, nous sommes donc engagés dans une crise généralisée du futur. Ce futur devient insaisissable, n'étant plus appréhendable par le seul tendanciel prévisible mais principalement tributaire de l'aléatoire chargé d'incertitude ; les innovations sont destinées de façon plus ou moins désordonnée à se succéder les unes les autres au gré de la plus ou moins grande incohérence du jeu des acteurs et de ce fait, empêchent comme l'a bien souligné A. Giddens ${ }^{2}$ la capacité de prévoir l'avenir.

De telles réflexions sur l'innovation et la temporalité sont particulièrement pertinentes pour comprendre la gestion par projet; nous nous trouvons là depuis un demi-siècle en présence de nouvelles formes de management qui expriment sous nos yeux les transformations du travail : ces formes entrent en opposition voire en conflit avec les logiques traditionnelles des métiers; dans ces derniers il s'agissait d'abord de valoriser les logiques de l'amont, celles d'un matériau donné sur lequel travailler à l'aide de procédures définies pour le transformer à travers la maîtrise d'un savoir-faire réglé. Avec le management de projet il y a au contraire un déplacement vers les logiques de l'aval. Celles-ci insistent sur la conception d'une nouvelle activité, d'un produit inédit, d'un service expérimental en ayant recours à l'appel d'offre, à la mission, au groupe de travail, à la commission ad hoc, au chantier. De ce point de vue le management par projet retrouve une parenté imprévue avec les temporalités du compagnonnage médiéval qui s'est organisé en intervenant de chantier en chantier.

5 Les logiques des métiers issues des corporations médiévales, réaménagées après l'abolition de ces corporations à la Révolution ont perdu progressivement de leur influence sans pour autant disparaître au cours de la montée de l'industrialisation. Les logiques industrielles centrées simultanément sur la fabrication en série, la division du travail, l'importance dévolue aux règles, aux objectifs, à la planification, ont imposé de nouveaux savoir-faire parfois en appauvrissant les savoir-faire anciens. Les métiers malmenés par l'ère de l'industrialisation qui fait la part belle aux professions vont entrer carrément en turbulence avec l'avènement du mode postindustriel de production. Aussi les logiques postmodernes du projet mettent-elles à mal ce qui nous reste des logiques de métiers en nous introduisant dans un environnement d'incertitude, de complexité et de flexibilité. Il ne s'agit plus de concevoir un objet en série déjà prédéterminé mais d'esquisser le profil d'un objet singulier à façonner. Avec ce projet postmoderne nous nous libérons d'anciennes temporalités qui comportaient malgré tout leur côté pervers, le temps continu, le temps répétitif, la durée indéterminée, le futur obsessionnel. Nous 
apprenons à côtoyer des temporalités inédites qui pour une part d'elles-mêmes nous introduisent dans de nouvelles formes de perversité, celles de l'urgence, des délais, de l'immédiateté.

6 L'impératif communicationnel propre à notre culture postmoderne va donner une grande extension aux méthodologies du projet ; celles-ci mettent désormais sur la place publique ce qui restait jusqu'ici encore du domaine privé, les intentions de l'auteur qui projette. Auparavant on pouvait innover, expérimenter à ses risques et périls, sans passer par l'obligation d'avoir à faire valider ses intentions ; aujourd'hui toute intention porteuse de projet, même la plus intime, doit donner lieu à validation par l'autorité de tutelle ou le commanditaire avant d'être mise en exécution. C'est principalement à travers les intentions explicitées, et donc soumises à validation, que se manifeste la dimension communicationnelle de tout projet. De ce point de vue il nous faut considérer les conduites de projet telles qu'elles se déploient sous nos yeux comme un sous-ensemble d'un ensemble plus vaste qui lui donne son sens, les pratiques communicationnelles.

\section{L'émergence des préoccupations autour de la qualité}

7 Le projet depuis qu'il a succombé à des effets de mode est certainement devenu un concept ambigu qui recouvre des pratiques elles-mêmes ambiguës tantôt innovantes, tantôt coercitives, tantôt émancipatrices, tantôt assujettissantes. La large utilisation qui est faite d'un tel concept nous fait de plus en plus développer des stratégies de prudence vis-à-vis de lui. En revanche, il ne semble pas en être de même pour le concept de qualité qui apparaît clair dans ses délimitations; ses prétentions ne sont qu'opératoires, anticipant et évaluant le résultat obtenu et le processus qui l'y conduit. De telles prétentions offrent apparemment toutes les garanties de concevoir des procédures naturellement transparentes. Qui serait donc contre la qualité? Pourtant une telle exigence de transparence tout à fait congruente avec la civilisation communicationnelle soucieuse de chasser l'équivoque semble néanmoins suspecte. Elle l'est dans sa façon de s'imposer d'emblée avec de plus en plus d'insistance. Il nous faut donc nous interroger pour savoir ce que cache cette volonté de transparence procédurale.

8 La qualité a finalement une moins longue histoire que le projet; ainsi nous n'avons pas besoin de remonter à la Renaissance pour en saisir les origines, comme c'est le cas avec le projet dans sa version architecturale. Nous pouvons appréhender la qualité dans ses premières manifestations au cœur de la société industrielle en pleine expansion, dans les années 1920, lorsque la compagnie américaine Bell Téléphone veut optimiser ses gains de production en s'interrogeant sur les critères de satisfaction de ses clients. Remarquons que dès ses débuts la qualité est associée, comme d'ailleurs d'une certaine façon le projet, à une logique de l'aval, ici à travers la liaison qualité-satisfaction des clients. Dans les entreprises françaises de production des années 1950 les services Qualité s'identifiaient au Bureau des Méthodes. Ces services étaient issus pour une large part de l'influence américaine qui déjà travaillait sur la qualité totale avec ses modes de gestion T.Q.M. (Total Quality Management) diffusée tant en Europe (Plan Marshall entre autres) qu'au Japon qui en arrivera à partir de cette exigence imposée par l'occupant américain d'alors à instituer les fameux cercles de qualité. Les années 1970 voient se déplacer la qualité de la production vers la consommation; la communication, par l'intermédiaire de la publicité et du marketing, exige un service et un produit qui répondent à des normes qualité ; son emprise grandissante va contribuer à développer le consumérisme c'est-à-dire renforcer 
les exigences du client. Quant aux années 1980-1990, elles redécouvrent les vertus des cercles de qualité et diffusent largement les nouvelles normes de qualité, ISO (International Standard Organisation) 9000, APNOR Association française de normalisation), Qualité totale et autres.

Ces dernières décennies nous introduisent donc de plus en plus dans une éthique de la qualité avec le zéro défaut, l'idéal de perfection, le prix de l'excellence. Elles favorisent un autre déplacement significatif, celui qui va de la production et de la consommation aux services, plus spécialement ceux ordonnés à la formation et au travail social ; c'est ainsi que depuis une dizaine d'années pour le moins, bon nombre d'associations en charge de formation permanente ou d'insertion sociale et professionnelle se sont rapidement saisies des démarches qualité de type industriel, sans doute à la recherche d'une forme de légitimité que seul le mimétisme avec le secteur industriel pouvait offrir. Dix ans après ce nouveau glissement, à l'orée des années 2000, la qualité tant du produit que du processus semble opérer un dérapage supplémentaire en direction de la qualification de l'agent qui initie les débats autour de la compétence. Quoi qu'il en soit, la qualité en tant que nouveau et subtil dispositif de contrôle génère des pratiques qui renforcent la place accordée à l'évaluation. Elle admet ici une nouvelle convergence avec les pratiques de projet lorsque ces dernières se focalisent sur les dispositifs de validations des intentions.

Il nous faudrait certes dater avec plus de précision les différentes générations qui ont véhiculé des formes spécifiques de qualité. Évoquons entre autres la qualité du produit au regard du coût de la non-qualité, la qualité comme maîtrise de la démarche de production de l'objet, la qualité comme expression des besoins du client en lien avec l'exigence de devoir le satisfaire, la qualité comme aptitude à satisfaire un usage... Nous nous en tiendrons ici uniquement au débat sur cette nouvelle culture qui introduit le recours à la qualité et sa signification.

\section{Équivoques autour des dispositifs de mise en place de la qualité}

11 La multiplication très rapide des démarches qualité ne peut que produire des équivoques comme ce fut le cas pour les conduites à projet; en effet si l'on associe la qualité à la situation exceptionnelle du zéro défaut, de l'excellence, de la qualité totale, transformer ce qui doit rester de l'ordre de l'exceptionnel en banalité ne va pas de soi. Certes l'avantage d'une telle appréhension de la qualité est de favoriser l'interchangeabilité des objets et services grâce à une meilleure lisibilité de ceux-ci ; ici encore la qualité rejoint le projet en devenant l'un des outils mis au service de la communication ; les critères qualité constituent un code pratique à partir duquel les organisations peuvent échanger sur leurs produits et les procédures qui permettent leur fabrication, en diminuant les équivoques. Mais réduire la qualité à cette interchangeabilité pose problème à plus d'un titre. Au-delà de cette réduction, mentionnons plusieurs équivoques qui gravitent autour du recours systématique aux démarches qualité :

Une première équivoque est le glissement sémantique que l'on fait subir à la qualité en la réduisant souvent à la quantité avec ses normes, ses indicateurs procéduraux et ses chartes. Ces différentes façons de quantifier la qualité introduisent aussi bien dans le processus de production (assurance de la qualité) que dans le produit terminal (contrôle de la qualité) des éléments de mesure réducteurs en transformant subrepticement la 
qualité en quantité. Certes la qualité a une connotation toujours positive au regard de la quantité. C'est la raison pour laquelle sans doute son emploi devient souvent un euphémisme pour cacher une façon de parler de quantité lorsque se trouvent par exemple évoqués la qualité totale et le zéro défaut. L'euphémisme vient de ce qu'on avance l'idée d'une qualité-différenciation pour mieux parler finalement d'une quantiténormalisation ${ }^{3}$. De ce point de vue on inverse un certain ordre de la nature; celle-ci n'a jamais fait dériver la quantité de la qualité : nous naissons hommes ou femmes mais pas un peu plus ou un peu moins hommes ou femmes. En revanche, Hegel l'a souligné en son temps, il est légitime de faire dériver la qualité de la quantité; en augmentant démesurément la température de l'eau ou en la baissant démesurément je change la nature de cette eau qui deviendra dans le premier cas vapeur, dans le second glace. Dans le débat actuel sur la qualité, cette dernière se métamorphose tant dans les usages verbaux que dans les pratiques en quantité, situation donc tout à fait insolite pour ne pas dire contre nature.

Une deuxième équivoque consiste dans la plupart des cas à subordonner la qualité à la satisfaction du client ${ }^{4}$. La qualité devient alors l'aptitude d'un produit, bien ou service à satisfaire les besoins spécifiques d'un ou plusieurs clients. Pourtant on sait bien que cette satisfaction du client est aléatoire, versatile pour ne pas dire capricieuse et en tout état de cause ne constitue que l'un des éléments de la situation. Répondre à la demande du client, par définition évolutive et instable, c'est se laisser piéger par un client susceptible de changer d'avis entre la commande et la livraison du produit ou du service. C'est pour le moins empêcher tout réponse innovante par l'une ou l'autre forme de projet.

Une troisième équivoque tend à assimiler de façon hyperbolique la qualité à l'excellence ; d'ailleurs l'expression qualité totale est suffisamment suggestive à cet effet ; la qualité se transforme alors en véritable mythe $\mathrm{e}^{5}$ au service d'une quête forcenée de la réussite complète par une mise en scène de l'excellence poussée jusqu'à l'extrême de l'absurde en faisant fi des imprévus. Dans le contexte des crises idéologiques liées à l'effacement des transcendances, la qualité s'offre ainsi à elle seule comme une nouvelle forme de transcendance sous couvert d'une éthique à portée de main.

Sans doute pourrait-on ajouter une quatrième équivoque, celle qui consiste à assimiler la qualité à une maitrise totale de la situation qui congédie l'aléatoire, l'incertitude de l'événement; cette quatrième équivoque nous renvoie à la tentation de l'efficience dans l'action, une efficience générée par l'impression de toute-puissance donnée par la raison instrumentale mais qui ne manquera pas de laisser échapper maints effets non prévus, voire pervers ${ }^{6}$.

16 Finalement à travers standardisation et normalisation, la qualité peut souvent se laisser réduire à un contrôle de conformité et relever de la vieille utopie scientiste de Taylor. Elle a toutefois pour avantage de mettre en place un système de codes facilitant la communication, l'échange d'informations entre systèmes de production, systèmes de services voire, au niveau international, systèmes culturels différents. De ce point de vue les systèmes qualité imposés par le commanditaire, s'ils gagnent en transparence, ne pourront que constituer une gêne programmatique, une entrave à la capacité d'innover du maître d'œuvre dont le projet va se trouver surveillé, suspecté, étroitement canalisé.

17 En revenant pourtant à l'origine étymologique de qualité, qui l'oppose à quantité, nous rencontrons alors de façon imprévue et inespérée une forte convergence avec les conduites de projet dans la mesure où la qualité ne concerne plus le particulier prescriptif 
mais le singulier inédit. En un mot, la qualité visait initialement la singularité de la réponse apportée compte tenu du contexte situationnel dans lequel elle s'inscrivait.

\section{La qualité, un espace multiréférentiel}

18 Finalement au-delà de ce rapide historique que nous venons d'effectuer et des ambiguïtés que nous avons soulignées, qu'est-ce que la qualité ? Cette dernière se laisse-t-elle réduire à ses usages organisationnels? Si nous revenons à l'origine étymologique de qualité, la qualitas des Latins, nous pouvons identifier la qualité à la manière d'être d'une chose, le mode d'être fondamental d'un objet; mais les manières et les modes peuvent changer; on n'est jamais bien sûr de les appréhender aussi facilement que nous le souhaiterions; aussi peut-on dire de la qualité qu'elle fait problème. Le problème vient pour une large part d'un glissement entre deux dimensions de la qualité ${ }^{7}$ :

une dimension qui particularise l'objet dans ses attributs attractifs observables extérieurement; la qualité d'une étoffe sera jugée à son tissage, la qualité d'un vin à la couleur de sa robe, la qualité d'un accueil au sourire prévenant de l'hôte ou de l'hôtesse... Ainsi en particularisant l'objet ou le service la qualité identifie cet objet ou ce service de façon relative et superficielle ; un critère choisi pour juger de la qualité pourra cacher un défaut, la couleur passée pour l'étoffe par exemple, le goût de bouchon pour le vin, l'hypocrisie de l'hôte ou de l'hôtesse pour l'accueil. Aussi cette dimension qui particularise doit se décliner obligatoirement au pluriel ; on parlera alors des qualités qui aident à spécifier l'objet; ces qualités sont considérées comme essentiellement relatives les unes par rapport aux autres.

20 - une dimension qui singularise; la qualité cesse alors d'être relative; elle cesse de récapituler un ensemble de particularités pour se poser comme différence essentielle; elle devient constitution de la chose qu'elle qualifie : la qualité de l'armoire rustique en face de laquelle je me trouve est d'être en chêne, la qualité de ce vêtement est d'être en coton, ou en tergal ou en laine. Nous passons ici du pluriel des particularités auxquelles se trouve associée la qualité au singulier d'une distinction considérée comme essentielle. Nous quittons en quelque sorte la variabilité des formes pour entrer dans l'invariance de l'essence. Nous passons d'une logique du particulier à une logique du singulier. D'une certaine façon c'est l'ensemble des qualités qui, toutes identifiées, contribuent à déterminer la spécificité d'un objet qui sera dit de qualité : cette armoire rustique en chêne clair avec une ornementation en pointes de diamant en élévation sur trois panneaux et comportant des pieds droits, qui l'apparentent au style Louis XIII.

21 Ainsi la qualité renvoie simultanément à un double registre, d'une part à un ensemble de particularités qui caractérisent un objet, d'autre part à ce qui fait la spécificité de l'objet dans sa singularité ; dans le premier cas on parlera des qualités de l'objet, dans le second cas d'un objet de qualité. Les normes qualité travaillent davantage sur le premier sens, le projet sur le second sens.

22 Ajoutons que ces deux dimensions que nous venons de définir gardent une signification subjective ou intersubjective essentielle. Elles font en effet l'objet de manipulations de la part des qualiticiens dans leur façon d'identifier certaines particularités ou une singularité en fonction des critères qu'ils se donnent; ils authentifient particularités et singularité en se basant toujours sur des appréciations. Il nous faut donc nous déplacer de l'objet qualifié vers le sujet qui qualifie et reconnaître que les critères de qualité sont à 
rechercher pour une part dans le jugement porté par l'évaluateur-qualiticien sur les choses; c'est un acte de jugement pouvant engager plus le qualiticien ou son système commanditaire que l'objet, le service ou la démarche évalués. À travers le jugement attaché à toute reconnaissance de qualité nous sommes là en présence de facteurs psychologiques et culturels essentiels. Les normes qualité dans leur mode d'invasion aujourd'hui interrogent autant les agents qui les édictent que les objets sur lesquels elles portent. Elles disent quelque chose sur une obsession culturelle du jour. De ce fait la signification subjective des jugements de qualité constitue une limite à la communication ; elle rend cette dernière en partie opaque.

\section{Projet et qualité et leurs modes d'articulation}

En prenant appui sur ce que nous venons d'argumenter jusqu'ici, nous pouvons entrevoir une double articulation entre la qualité et le projet en fonction des deux sens contrastés que la qualité revêt, que nous avons mis en évidence.

D'une part, dans la mesure où la qualité se définit en termes de normes et de spécifications, elle renvoie au commanditaire et à son programme qui fixent un niveau d'exigences ; c'est ce niveau que le concepteur du projet doit satisfaire lorsqu'il répond à une commande; la validation du projet par le commanditaire au terme du processus de conception, l'évaluation du projet à l'issue du travail de réalisation pourront dire l'une et l'autre si les exigences sont respectées à travers une évaluation de conformité.

La moitié du travail autour de la qualité est ainsi faite lorsque le commanditaire a pu préciser sa demande et expliciter ses critères qualité à l'auteur du projet en position de répondre à un appel d'offres. Les critères qualité sont répertoriés par le commanditaire pour que le client soit satisfait. C'est à partir de ces critères que l'auteur du projet va élaborer sa proposition innovante. L'autre moitié du travail lié à la qualité est alors à faire et se trouve en conséquence entre les mains du concepteur du projet ou de l'équipeprojet que ce dernier anime. La démarche de projet est à conduire en cherchant à apporter une réponse singulière à la commande ; pour ce faire, elle se donne à elle-même ses propres exigences d'innovation, de créativité. Elle cherche ainsi à effectuer un travail de qualité en élaborant une réponse tout à fait inédite au regard de la commande passée et de la situation qui lui est liée, tout en respectant les critères qualité de cette commande.

L'auteur du projet au regard du travail de qualité qui lui incombe est donc finalement confronté à un double ensemble d'exigences, des exigences externes qui doivent lui faire satisfaire les normes qualité, des exigences internes qui lui font produire un travail de qualité. Autant les premières dans leur souci d'objectivité seront davantage sujets à communication et diminueront dans le meilleur des cas les équivoques entre commanditaire et concepteur, autant les secondes vont introduire une certaine forme d'opacité dans la communication, le travail de qualité renvoyant à une appréciation davantage multidimensionnelle et subjective. Mais toute commande n'est-elle pas faite d'un troublant paradoxe qui associe des lambeaux de transparence et des lambeaux d'opacité ? Ce balancement incessant entre transparence et opacité n'est-il pas par ailleurs une caractéristique fondatrice de notre actuelle culture communicationnelle? 


\section{Norme qualité du commanditaire et projet de qualité du concepteur}

27 La culture de la qualité semble actuellement reposer sur trois socles, le premier concerne la rationalisation instrumentale qui fait abuser des critères de qualité souvent transformés en indicateurs quantitatifs à prendre en compte. Ce premier socle présente toutefois l'avantage de rendre plus compréhensibles et maitrisables les procédures utilisées. Le second depuis l'origine des préoccupations autour de la qualité mais de façon très amplifiée aujourd'hui touche l'hédonisme à travers le postulat de la satisfaction du client; ce client dans la prise en charge qu'il sollicite de ses besoins, entend par l'objet désiré et possédé ou le service sollicité éprouver une forme de jouissance, cette jouissance propre à tout consommateur. Le troisième socle est plus proprement le socle communicationnel avec cette obsession de transparence, celle obtenue par les différentes certifications visant à instituer une nouvelle langue débarrassée de toute forme d'équivocité. Ce socle communicationnel permet de comparer les objets entre eux en fonction de leur degré de labellisation et à partir de leurs spécifications. Il donne la possibilité aux constructeurs de communiquer en utilisant les mêmes codes, de se mesurer dans une logique concurrentielle voire de s'échanger des informations; il offre enfin aux procédures les moyens d'un classement, d'une hiérarchisation pour pouvoir déterminer chez les opérateurs concernés des types de compétence.

C'est sur ces trois socles constitutifs des référentielles qualités, l'instrumentalisation, l'hédonisme, la communication que devra s'ébaucher à travers l'une ou l'autre forme de projet, une réponse qui en tout état de cause ne saurait échapper à l'exigence d'être de qualité.

Cette tension à aménager entre ces deux acceptions du concept de qualité au sein des pratiques managériales est, selon nous, la seule façon d'éviter que nos pratiques tombent dans la culture du contrôle chaque fois qu'elles recourent à la qualité et au projet; ces derniers en effet possèdent ce trait commun lorsqu'ils sont largement diffusés, d'engendrer de nouvelles et subtiles formes de coercition. En revanche, restituer les exigences du commanditaire dans ses recommandations autour de la qualité, préserver l'espace créateur de l'acteur qui projette en lui reconnaissant la légitimité d'effectuer une contribution de qualité, c'est là donner à notre société communicationnelle la chance de rester interactive et créative.

\section{NOTES}

1. Gestion de ou par projet, management de ou par projet sont des formulations équivalentes pour désigner un mode d'expérimentation confié à un groupe, le groupe-projet, à qui se trouve confiée une mission pour une durée déterminée avec des moyens déterminés.

2. Cf. en traduction, les conséquences de la modernité, Paris, L'Harmattan, 1994 
3. Cette ambiguïté au sein de la qualité entre la standardisa-bon et la différenciation est bien mise en évidence par D. Segrestin, L'entreprise à l'épreuve des normes de marché. Revue française de sociologie, XXXVIII, 1997, pp. 553-585.

4. C'est du moins comme cela que I. Kelada la définit dans son ouvrage, Comprendre et réaliser la qualité totale, Editions Quafec, 1995.

5. Sur ce mythe $c f$. les différentes contributions parues dans la revue Autrement, L'excellence, une valeur pervertie, 1987.

6. Sur une critique de la rationalité instrumentale, voir la contribution récente de la Revue internationale de Psychosociologie dans son numéro spécial sur La résistible emprise de la rationalité instrumentale, III, 8, 1997.

7. Ces deux dimensions nous ont été inspirées par le travail fort suggestif de J-Ch. Torres, Hexis et poios. essai d'une analyse conceptuelle de la qualité, Éducation permanente, 126,1,1996.

\section{RÉSUMÉS}

Notre civilisation communicationnelle a vu l'émergence concomitante, ces dernières années, des préoccupations autour du projet et de la qualité. En situant les deux concepts multi-référentiels dans leur contexte historique d'apparition, nous nous interrogeons sur les convergences et les paradoxes susceptibles de les associer. Nous cherchons à voir en quoi ils constituent deux bons analyseurs des pratiques communicationnelles renvoyant deux exigences situées au cœur des pratiques organisationnelles, la norme-qualité du commanditaire d'un côté, le projet de qualité du concepteur de l'autre. Or ces exigences sont souvent confondues, prises l'une pour l'autre, faisant de la qualité et du projet, des concepts flottants qui rendent souvent équivoque la compréhension de processus communicationnels en jeu.

Our communicative civilisation has withnessed the simulations emergence, over the last few years, of concerns about the project and the quality. By replacing the two multireferential concepts within the historical context of their emergence, we will deal with the question of the convergences and the paradoxes which are likely to reunite them. We will try to find out in what way they are both the right analysers of communicative habits, the quality standard of the sponsor on the one hand, the designer's quality project on the other hand. Now these requirements are often mistaken, turning the quality and the project into floating concepts which foster ambiguity in the understanding of the communicative process at stake.

\section{INDEX}

Mots-clés : projet, qualité, communication

\section{AUTEUR}

\section{JEAN-PIERRE BOUTINET}

Jean-Pierre Boutinet est professeur à l'UCO-IPSA à Angers, directeur de l'Institut de Recherche Fondamentale et Appliquée d'Angers. Il. est par ailleurs professeur associé à 
l'Université de Sherbrooke (Canada). Auteur entre autres d'Anthropologie du Projet et de L'Immaturité de la vie adulte aux Presses Universitaires de France. Il a aussi rédigé deux «Que sais-je ?» : Psychologie des Conduites à Projet, Psychologie de la Vie Adulte. 